\title{
Heterogeneous approach to nebulization of antimicrobial agents in mechanically ventilated adults in a German tertiary care hospital: a cross-sectional survey
}

\author{
Frieder Pfäfflin ${ }^{1,2}$ (D) Miriam Stegemann ${ }^{1,2} \cdot$ Norbert Suttorp $^{1} \cdot$ Alexander Uhrig $^{1} \cdot$ Stephan Achterberg ${ }^{1,2}$
}

Received: 2 June 2020 / Accepted: 24 August 2020 / Published online: 3 September 2020

(C) The Author(s) 2020

\begin{abstract}
There is lack of standardization of practices and limited evidence on efficacy and safety of nebulization of antimicrobials. We sought to determine inhalation practices in one tertiary care hospital by performing a cross-sectional survey. Eleven adult ICUs were included in the analysis. Three units followed established protocols. Ventilation circuit filters were exchanged at least daily in all but one units. Dosages of aminoglycosides and CMS depended on indication and unit. Nebulization of antimicrobials was generally regarded as safe and efficacious. Our data indicate that approach to nebulization of antimicrobials may be heterogeneous even in a single center.
\end{abstract}

Keywords Nebulization $\cdot$ Invasive ventilation $\cdot$ Antimicrobial $\cdot$ AMS $\cdot$ Heterogeneous $\cdot$ Pneumonia

\section{Introduction}

Guidelines for nebulization of antimicrobial agents vary. The German guidelines for treatment of nosocomial pneumonia indicate that inhaled administration of antibiotics should be considered in addition to systemic antibiotic therapy in patients with multidrug-resistant organisms (MDRO), which are only susceptible to polymyxins and/or aminoglycosides [1]. Contrarily, the European Society of Clinical Microbiology and Infectious Diseases (ESCMID) does not support the use of nebulization of antibiotics due to a weak level of evidence for their efficacy and the high potential for underestimated risks of adverse events [2]. Furthermore, recent international consensus guidelines for the optimal use of polymyxins advocate the use of adjunctive

Electronic supplementary material The online version of this article (https://doi.org/10.1007/s10096-020-04017-0) contains supplementary material, which is available to authorized users.

Frieder Pfäfflin

frieder.pfaefflin@charite.de

1 Department for Infectious Diseases and Respiratory Medicine, Charité - Universitätsmedizin Berlin, corporate member of Freie Universität Berlin, Humboldt-Universität zu Berlin, and Berlin Institute of Health, Augustenburger Platz 1, 13353 Berlin, Germany

2 Antibiotic Stewardship, Charité - Universitätsmedizin Berlin, corporate member of Freie Universität Berlin, Humboldt-Universität zu Berlin, and Berlin Institute of Health, Berlin, Germany polymyxin aerosol therapy in patients with extensively resistant gram-negative nosocomial or ventilator-associated pneumonia (VAP) who require intravenous polymyxin [3]. In this journal, Alves et al. reported the results of an international cross-sectional survey on nebulization of antimicrobial agents in mechanically ventilated adults. They found considerable heterogeneity of practice among participating intensive care units (ICU) from different continents [4]. However, little is known about different practices within a single institution. During audits, members of the antimicrobial stewardship (AMS) team of Charité Universitätsmedizin Berlin had the impression that the approach to nebulization of antimicrobial agents differed among ICUs. We therefore sought to determine inhalation practices systematically in our institution by performing a cross-sectional survey.

\section{Methods}

Charité-Universitätsmedizin Berlin is a large tertiary care hospital with more than 3000 in-patient beds and 25 ICUs on three campuses. As of May 2020, the AMS team comprised four physicians (one microbiologist and three infectious disease physicians) and two clinical pharmacists. Audits with direct feedback are conducted on all wards with high consumption of antimicrobial agents on a regular basis. Within audits, one or two specialist physicians who act as focal persons for AMS represent each ICU. We addressed all 18 adult ICUs. Wards with less than five cases per month were 
considered ineligible. The survey was built on the SANEME 2 study protocol and adapted to the local situation [5]. Questions focused on key aspects of nebulized antimicrobial agents, which were (1) indications, (2) agents used, (3) dosages, and (4) devices and ventilation settings (see supplementary material). We targeted one specialist physician per ICU who was interviewed by two AMS team members (FP and SA). If the physician was unaware of practices, he was free to ask staff from the unit for assistance. Responses were analyzed by using descriptive statistics.

\section{Results}

Seventeen out of 18 ICUs responded. Six ICUs were excluded as they estimated to treat less than five patients per month with nebulized antimicrobials. The median number of beds of the remaining ICUs was 16 (range 10 to 24). Vibrating mesh nebulizers, ultrasonic nebulizers, and jet nebulizers were used in $6 / 11$ wards (54.5\%), 4/11 wards (36.4\%), and 1/11 wards (9.1\%), respectively. The filter placed on the expiratory limb of ventilation circuits was changed daily in 9/11 wards (81.8\%), weekly in $1 / 11$ wards $(9.1 \%)$, and after every nebulization in $1 / 11$ wards (9.1\%). Three wards (27.2\%) followed established protocols for administration of nebulized antimicrobials. Bronchodilation before nebulization was conducted always in 5/11 wards (45.5\%), as needed in $4 / 11$ wards $(36.4 \%$ ), and never in $2 / 11$ wards $(18.2 \%)$. Two units $(18.2 \%)$ increased tidal volume for nebulization of antimicrobials. All other ventilator settings in all of the units remained unchanged. Gentamicin, tobramycin, and colistin methanesulfonate (CMS) were used preferably, while one ward also used liposomal amphotericin B. Prescribed dosages differed depending on indication and ward. Dosages ranged for gentamicin from $40 \mathrm{mg} \mathrm{q} 24 \mathrm{~h}$ to $80 \mathrm{mg}$ q $8 \mathrm{~h}$, for tobramycin from $80 \mathrm{mg}$ q24h to $300 \mathrm{mg} \mathrm{q} 12 \mathrm{~h}$, and for CMS from $1 \mathrm{MU}$ q24h to 2 MU q8h (Table 1). When nebulized antimicrobials were used for treatment of VAP, they were always combined with systemic antibiotics. Nebulization was regarded as safe and very safe in 5/11 (45.5\%) and 6/11 wards (54.5\%), respectively. All wards regarded efficacy as good.

\section{Discussion}

Our data indicate that even within one hospital, the approach to nebulization of antimicrobial drugs may be heterogeneous. This may partly be explained by the organizational structure of Charité - Universitätsmedizin Berlin, where ICUs are run by different departments on three different campuses. However, the range in practices may also reflect current national and international guidelines, which give conflicting advice [1-3]. Only three out of eleven ICUs followed established protocols for administration of nebulized antibiotics. Physicians on these wards in particular had extensive experience with inhaled antimicrobials. In one ICU, which is a certified weaning center, inhalation of low dose
Table 1 Use of nebulized antimicrobials for specified indications

\begin{tabular}{|c|c|c|c|c|}
\hline \multirow[t]{2}{*}{ Drug } & \multirow[t]{2}{*}{ Prescribed dosage } & \multicolumn{3}{|c|}{$\begin{array}{l}\text { No of units administering nebulized } \\
\text { antimicrobials for specified indications }\end{array}$} \\
\hline & & $\begin{array}{l}\text { Prophylaxis of VAT } \\
\text { and/or VAP }\end{array}$ & Treatment of VAT & Treatment of VAP \\
\hline $\begin{array}{l}\text { Liposomal } \\
\quad \text { amphotericin B }\end{array}$ & $\mathrm{np}$ & & & 1 \\
\hline \multirow[t]{5}{*}{ CMS } & $1 \mathrm{MIU}$ q24h & 2 & & \\
\hline & $1 \mathrm{MIU}$ q12h & & 1 & 2 \\
\hline & $1 \mathrm{MIU}$ q8h & 2 & & \\
\hline & 2 MIU q12h & & & 2 \\
\hline & $2 \mathrm{MIU}$ q8h & & & 2 \\
\hline \multirow[t]{3}{*}{ Gentamicin } & $40 \mathrm{mg} \mathrm{q} 24 \mathrm{~h}$ & 1 & & 1 \\
\hline & $80 \mathrm{mg} \mathrm{q} 24 \mathrm{~h}$ & 2 & & \\
\hline & $80 \mathrm{mg} \mathrm{q} 8 \mathrm{~h}$ & & 2 & 2 \\
\hline \multirow[t]{5}{*}{ Tobramycin } & $80 \mathrm{mg} \mathrm{q} 24 \mathrm{~h}$ & 5 & & 1 \\
\hline & $80 \mathrm{mg} \mathrm{q} 12 \mathrm{~h}$ & 3 & 1 & 1 \\
\hline & $80 \mathrm{mg} \mathrm{q} 8 \mathrm{~h}$ & & 3 & 5 \\
\hline & $80 \mathrm{mg} \mathrm{q6}-8 \mathrm{~h}$ & & 1 & 1 \\
\hline & $300 \mathrm{mg} \mathrm{q} 12 \mathrm{~h}$ & 2 & & 3 \\
\hline
\end{tabular}

$C M S$ colistin methanesulfonate, $n p$ not provided, No number, VAT ventilator-associated tracheobronchitis, VAP ventilator-associated pneumonia

${ }^{\text {a }}$ Sum of units may be greater than total number of units as some units use several nebulized antimicrobials for a given indication 
gentamicin (i.e. $40 \mathrm{mg}$ q24h) is prescribed to all invasively ventilated patients to prevent episodes of VAP. Gentamicin may be replaced by other antibiotics if the colonizing flora is resistant to gentamicin. In case of VAP, other inhaled antibiotics are administered at higher dosage (usually tobramycin $80 \mathrm{mg} \mathrm{q} 8 \mathrm{~h}$ ) and systemic antibiotic treatment is added. Treatment with inhaled gentamicin is resumed after antibiotic therapy for VAP has ended. Physicians of this ward consider this strategy as safe and efficacious; however, further study is necessary to prove or refute its benefit. We found substantial uncertainty regarding nebulization of antimicrobials on most units. In some wards, physicians were unaware of the device used for nebulization and several physicians asked for recommendations on how to approach inhaled antimicrobials. Nonetheless, protocols for filter exchange were in place on all wards and filters were exchanged at least daily in 10 out of 11 units. In the international survey by Alves et al., daily filter changes were carried out in 83 of 216 (38.4\%) units [4]. The high percentage of daily filter exchange in our hospital may contribute to the perception of all respondents that nebulization of antibiotics was safe as adverse events in the literature have been linked to obstruction of the expiratory filter [6]. However, respondents in the study by Alves et al. commonly described bronchospasm, cough, and a moderate decrease in oxygen saturation [4]. All these adverse events may occur independently of frequent filter exchanges. Inhalation of antimicrobial drugs was regarded unanimously as efficacious although all respondents admitted that this was merely a subjective estimation. AMS programs face difficulties in giving sound advice on nebulization of antimicrobials. Limited evidence and risk of adverse events caution against liberate administration. On the other hand, there may be a role in treating MDROs and a potential in preventing episodes of VAP. Although the indications for nebulized antibiotics are debatable, technical requirements should be followed to minimize risks of treatment failure and adverse events [7]. The observed heterogeneity of practices in our institution might constitute an argument for institutional-wide standardized procedures and standardized guidelines in general.

Funding Open Access funding provided by Projekt DEAL.

\section{Compliance with ethical standards}

Conflict of interest The authors declare that they have no conflict of interest.

Ethical approval Institutional ethical review board approval was not required as this analysis was based on a clinical practice survey.

Informed consent This analysis does not contain patient data; therefore, informed consent was not required

Open Access This article is licensed under a Creative Commons Attribution 4.0 International License, which permits use, sharing, adaptation, distribution and reproduction in any medium or format, as long as you give appropriate credit to the original author(s) and the source, provide a link to the Creative Commons licence, and indicate if changes were made. The images or other third party material in this article are included in the article's Creative Commons licence, unless indicated otherwise in a credit line to the material. If material is not included in the article's Creative Commons licence and your intended use is not permitted by statutory regulation or exceeds the permitted use, you will need to obtain permission directly from the copyright holder. To view a copy of this licence, visit http://creativecommons.org/licenses/by/4.0/.

\section{References}

1. Dalhoff K, Abele-Horn M, Andreas S, Deja M, Ewig S, Gastmeier P, Gatermann S, Gerlach H, Grabein B, Heussel CP, Hoffken G, Kolditz M, Kramme E, Kuhl H, Lange C, Mayer K, Nachtigall I, Panning M, Pletz M, Rath PM, Rohde G, Rosseau S, Schaaf B, Schreiter D, Schutte H, Seifert H, Spies C, Welte T (2018) C. Unter Mitwirkung der folgenden Wissenschaftlichen Fachgesellschaften und Institutionen: Deutsche Gesellschaft fur, V. Deutsche Gesellschaft fur Innere Medizin e, N. Deutsche Gesellschaft fur Internistische Intensivmedizin und, V. Deutsche Sepsis-Gesellschaft e, K.-I. und Robert, [Epidemiology, diagnosis and treatment of adult patients with nosocomial pneumonia - update 2017 - S3 Guideline of the German Society for Anaesthesiology and Intensive Care Medicine, the German Society for Infectious Diseases, the German Society for Hygiene and Microbiology, the German Respiratory Society and the Paul-Ehrlich-Society for Chemotherapy, the German Radiological Society and the Society for Virology], Pneumologie 72(1) 15-63

2. Rello J, Sole-Lleonart C, Rouby JJ, Chastre J, Blot S, Poulakou G, Luyt CE, Riera J, Palmer LB, Pereira JM, Felton T, Dhanani J, Bassetti M, Welte T, Roberts JA (2017) Use of nebulized antimicrobials for the treatment of respiratory infections in invasively mechanically ventilated adults: a position paper from the European Society of Clinical Microbiology and Infectious Diseases. Clin Microbiol Infect 23(9):629-639

3. Tsuji BT, Pogue JM, Zavascki AP, Paul M, Daikos GL, Forrest A, Giacobbe DR, Viscoli C, Giamarellou H, Karaiskos I, Kaye D, Mouton JW, Tam VH, Thamlikitkul V, Wunderink RG, Li J, Nation RL, Kaye KS (2019) International consensus guidelines for the optimal use of the polymyxins: endorsed by the American College of Clinical Pharmacy (ACCP), European Society of Clinical Microbiology and Infectious Diseases (ESCMID), Infectious Diseases Society of America (IDSA), International Society for Anti-infective Pharmacology (ISAP), Society of Critical Care Medicine (SCCM), and Society of Infectious Diseases Pharmacists (SIDP), Pharmacotherapy 39(1) 10-39

4. Alves J, Alp E, Koulenti D, Zhang Z, Ehrmann S, Blot S, Bassetti M, Conway-Morris A, Reina R, Teran E, Sole-Lleonart C, Ruiz-Rodriguez M, Rello J, S.-. Investigators (2018) Nebulization of antimicrobial agents in mechanically ventilated adults in 2017: an international cross-sectional survey. Eur J Clin Microbiol Infect Dis 37(4):785-794

5. Rello J, Ruiz-Rodriguez M, Zhang Z (2017) 2017 Global survey on nebulization of antimicrobial agents in mechanically ventilated patients - SANEME 2 study protocol. J Emerg Crit Care Med 1(2)

6. Lu Q, Yang J, Liu Z, Gutierrez C, Aymard G, Rouby JJ, G. Nebulized Antibiotics Study (2011) Nebulized ceftazidime and amikacin in ventilator-associated pneumonia caused by Pseudomonas aeruginosa. Am J Respir Crit Care Med 184(1):106-115

7. Rello J, Rouby JJ, Sole-Lleonart C, Chastre J, Blot S, Luyt CE, Riera J, Vos MC, Monsel A, Dhanani J, Roberts JA (2017) Key considerations on nebulization of antimicrobial agents to mechanically ventilated patients. Clin Microbiol Infect 23(9):640-646

Publisher's note Springer Nature remains neutral with regard to jurisdictional claims in published maps and institutional affiliations. 